\title{
Functional near-infrared spectroscopy (fNIRS) brain imaging of multi-sensory integration during computerized dynamic posturography in middle-aged and older adults
}

\author{
Chia-Cheng Lin ${ }^{1,4}$, Jeffrey W. Barker ${ }^{3}$, Patrick J. Sparto ${ }^{1,2}$, Joseph M. Furman ${ }^{1,2}$, and \\ Theodore J. Huppert ${ }^{3}$ \\ ${ }^{1}$ Department of Physical Therapy, University of Pittsburgh, Pittsburgh, PA, USA \\ ${ }^{2}$ Department of Otolaryngology, University of Pittsburgh, Pittsburgh, PA, USA \\ ${ }^{3}$ Department of Radiology, University of Pittsburgh, Pittsburgh, PA, USA \\ ${ }^{4}$ Department of Physical Therapy, East Carolina University, Health Sciences Building, 2405D, Mail \\ Stop 668, Greenville, NC 27834, USA
}

\begin{abstract}
Studies suggest that aging affects the sensory re-weighting process, but the neuroimaging evidence is minimal. Functional Near-Infrared Spectroscopy (fNIRS) is a novel neuroimaging tool that can detect brain activities during dynamic movement condition. In this study, fNIRS was used to investigate the hemodynamic changes in the frontal-lateral, temporal-parietal, and occipital regions of interest (ROIs) during four sensory integration conditions that manipulated visual and somatosensory feedback in 15 middle-aged and 15 older adults. The results showed that the temporal-parietal ROI was activated more when somatosensory and visual information were absent in both groups, which indicated the sole use of vestibular input for maintaining balance. While both older adults and middle-aged adults had greater activity in most brain ROIs during changes in the sensory conditions, the older adults had greater increases in the occipital ROI and frontal-lateral ROIs. These findings suggest a cortical component to sensory re-weighting that is more distributed and requires greater attention in older adults.
\end{abstract}

\section{Keywords}

Functional near-infrared spectroscopy (fNIRS); Aging; Sensory re-weighting

\section{Introduction}

Human balance control is reliant on three classes of sensory inputs: somatosensation, vision, and vestibular sensation (Nashner and Berthoz 1978). Recent studies have suggested that postural control is a dynamic process which involves re-weighting the three sensory inputs to adapt environmental changes (Peterka 2002; Peterka and Loughlin 2004; Mahboobin et al. 2005; O'Connor et al. 2008). In addition, studies have shown that the sensory inputs may 
be used to varying degrees during different life stages (Lee and Aronson 1974; Nashner and Berthoz 1978; Woollacott et al. 1987; Woollacott and Shumway-Cook 1990).

Aging is one of the factors that may affect sensory re-weighting (Laurienti et al. 2006; O'Connor et al. 2008; Faraldo-Garcia et al. 2012). Several studies have used computerized dynamic posturography (Nashner et al. 1982) or other similar systems (Allum et al. 2002) to investigate how age affects sensory re-weighting during postural control (Cham et al. 2007; O'Connor et al. 2008; Faraldo-Garcia et al. 2012). The demonstrations of the evidence of sensory re-weighting in these studies were based primarily on biomechanical measures while performing dynamic balance tasks. However, other studies have tried to investigate the sensory re-weighting process from a top-down approach, using external cortical stimulation, such as transcranial magnetic stimulation (TMS) or neuroimaging tools, such as functional magnetic resonance (fMRI). The TMS studies have shown that the motor cortex is involved in balance control and that its inhibitory activity decreases with age (Taube et al. 2007; Papegaaij et al. 2014b). Using fMRI neuroimaging studies, it has been shown that stance and locomotion rely on multiple cortical areas activity (Zwergal et al. 2012). Zwergal et al. suggested that activation of some cortical areas, such as visual cortical areas, were independent of age, while other cortical areas may increase activation (i.e. decrease inhibition) with age, such as multi-sensory vestibular cortical areas (Zwergal et al. 2012). However, fMRI is not able to measure brain activities during dynamic postural balance conditions.

Functional near-infrared spectroscopy (fNIRS) is a noninvasive optical brain imaging method that detects cortical activation in a natural environment (Faraldo-Garcia et al. 2012). Several studies have suggested that fNIRS has high temporal and spatial correlation with MRI during brain hemodynamic changes (Huppert et al. 2006a, b). Applying fNIRS to study balance control has shown that the different cortical areas play different roles in postural control (Mihara et al. 2008; Karim et al. 2012, 2013a; Huppert et al. 2013). The prefrontal cortex has been associated with dual-task stepping choices (Huppert et al. 2013) and visuospatial attention cues during perturbations in the anteroposterior direction (Mihara et al. 2008). Studies also confirmed the regions involved with multi-sensory integration using fNIRS (Karim et al. 2012, 2013a). However, how age affects the cortical activation during sensory integration tasks has not been investigated. In this study, fNIRS was used to measure cortical blood oxygenation changes during four different sensory integration conditions in middle-aged and older healthy adults.

\section{Methods}

\section{Experimental participants}

Fifteen healthy middle-aged adults (MA, 5M/10F, age $46 \pm 11$ years) and 15 healthy older adults (OA, 8M/7F, age $73 \pm 5$ years) participated in this study. All participants were righthanded. After providing informed consent, participants had screening tests performed to exclude those with vestibular, balance, and mobility impairments. The screening tests included a general neurological examination, a caloric vestibular function test (Jongkees 1948), and the Functional Gait Assessment (Whitney et al. 2004; Wrisley et al. 2004) by a 
physical therapist. This study protocol was approved by the University of Pittsburgh Institutional Review Board.

\section{fNIRS instrumentation}

The principle of fNIRS for brain imaging is based on the different absorption rates of oxyand deoxy-hemoglobin at two different wavelengths of light (690 and $830 \mathrm{~nm})$. The wavelengths may vary in different instruments. Source-detector pairs are basic elements to construct the brain spatial map. The sources are used to deliver the two different wavelengths and the detectors measure the attenuation of light due to absorption and scattering by the intervening tissues of the head. When the brain cortex evokes activity, the regional change in blood flow alters the concentration of oxy- and deoxy-hemoglobin which changes the absorption rate of the two different wavelengths. The modified Beer-Lambert law is used to convert the changes in light intensity to the changes in hemoglobin concentration. Thus, the brain spatial map can be made by spatially arranging the source and detector pairs on the head so the region of interest can be approximated (Boas et al. 2004).

In this study, a 30-channel continuous wave fNIRS instrument, consisting of 18 detectors and 12 sources, (CW6 real-time system; TechEN Inc, Milford, MA, USA) was used in this study. This fNIRS instrument has been used in previous studies (Abdelnour and Huppert 2009; Karim et al. 2012, 2013a, b). A three-piece cap with the optodes embedded in it was used to record the hemodynamic changes bilaterally in the frontal-lateral, temporal-parietal, and occipital regions (Fig. 1). Two different wavelengths of light, 690 and $830 \mathrm{~nm}$, were emitted by the 12 sources to assess the changes of both oxy- and deoxy-hemoglobin concentration in the brain cortex. There were 11 source-detector pairs (four sources and six detectors) over each lateral hemisphere and 12 source-detector pairs (four sources and six detectors) over the occipital region (Fig. 1). Each pair was arranged in a nearest neighbor geometry with $3 \mathrm{~cm}$ spacing. FNIRS data were sampled at $4 \mathrm{~Hz}$. The frontal-lateral region of interest (ROI) consisted of the two most anterior sources on each side and their nearestneighbor detectors and targeted activity in the executive function/attention regions; the temporal-parietal ROI was centered over the superior temporal gyrus and thus targeted activity in the multi-sensory vestibular cortex; the occipital ROI targeted activity in the visual cortex. Custom data acquisition software was used to record the hemodynamic changes in each source-detector pair (Abdelnour and Huppert 2009).

\section{Procedures}

All participants performed four trials of standing on a NeuroCom posturography platform (Natus ${ }^{\circledR}$, Clackamas, OR, USA) so that the visual and somatosensory inputs could be modified, while fNIRS signals were recorded. An $\mathrm{A}_{1}-\mathrm{B}-\mathrm{A}_{2}$ block design was implemented in this study. Table 1 describes the test conditions of the four trials. The sensory modifications involved changing either visual input [Eyes open in light (EO) and dark (EOD)] or somatosensory input [Fixed or Sway-referenced (SR) platform]. The EOD condition was accomplished by having the participants keep their eyes open while wearing darkened goggles. Each trial consisted of a change from greater sensory input to reduced sensory input. Each sub-block lasted $40 \mathrm{~s}$. All the participants performed the four trials one 
time, in random order. The stimuli markers were recorded in the fNIRS data acquisition when each block started to synchronize the postural data with fNIRS data.

\section{fNIRS data analysis}

The analysis of fNIRS data can be found in several previous papers (Huppert et al. 2009; Ye et al. 2009; Karim et al. 2013a). In general, the fNIRS time series data were converted to optical density followed by conversion to oxyhemoglobin concentration, [HbO] and deoxyhemoglobin concentration $[\mathrm{Hb}]$ via the modified Beer-Lambert law (Cope et al. 1988). The mean of the fNIRS signal during the test condition (B) relative to the mean of the fNIRS signal during the pre-baseline condition ( $\left.\mathrm{A}_{1}\right)$ was estimated using a regression model and computed using an autoregressive model, prewhitened iterative reweighted least squares (AR-IRLS) algorithm that is robust to artifacts introduced by subject motion and serial correlations introduced by systemic physiology (Barker et al. 2013). Based on our previous work (Karim et al. 2013a, b), we used a hemodynamic response function (HRF) that had an elongated return to baseline after the termination of the test condition. The relative signal change was computed on a per subject basis, in which the results were used for a second, group-level analysis. Group level analysis was performed via a linear mixed effects model that included random intercept terms for each subject to model within-subject correlations (Abdelnour and Huppert 2010). The group level model was estimated per channel using the built-in MATLAB 2014b package for estimating linear mixed effects models (Mathworks, Natick, MA, USA). The fNIRS measurement positions were registered to an anatomical atlas brain (Colin27) (Holmes et al. 1998) using a custom registration algorithm based on the international 10-20 coordinate system (Tsuzuki and Dan 2014).

For each channel, estimates of the regression coefficients, $\beta$, for $[\mathrm{HbO}]$ and $[\mathrm{Hb}]$, as well as the standard error, were computed for each subject group (Older Adults, Middle-aged Adults), and each trial. T-tests were used to determine if the regression coefficient differed from 0 , using the false discovery rate method to adjust the $p$ value $\left(p_{\text {FDR }}<0.05\right)$. Finally, a region-of-interest (ROI) analysis using all channels within each of five ROIs (occipital, right frontal-lateral, right temporal-parietal, left frontal-lateral, left temporal-parietal) was performed to test if there was a difference in $[\mathrm{HbO}]$ and $[\mathrm{Hb}]$ between subject groups, using $p_{\mathrm{FDR}}<0.05$.

\section{Postural sway data}

Center of pressure (COP) data were recorded from the NeuroCom posturography force plate. The root-mean-square (RMS) of the COP data in the anteroposterior direction was calculated for the two baseline conditions and the test condition for each trial. For each trial, a repeated measures ANOVA was used to examine the between subject effects of group (MA and $\mathrm{OA}$ ) and within subject effect of block $\left(\mathrm{A}_{1}, \mathrm{~B}\right.$, and $\left.\mathrm{A}_{2}\right)$ and their interaction. Post-hoc tests of pairwise differences were examined if the main effect of block or its interaction with group were significant, using a Sidak adjustment for multiple comparisons. The level of significance was $\alpha=0.05$. 


\section{Results \\ fNIRS responses}

Changes in the oxy-hemoglobin responses during the test condition compared with the prebaseline condition are illustrated in Fig. 2 for MA and OA. Qualitatively, [Hb] responses mirrored the $[\mathrm{HbO}]$ results, but with lesser magnitude of changes. As a result, this article will focus on $[\mathrm{HbO}]$ responses. During Trial 1 , when the somatosensory information was degraded while visual and vestibular information was retained, MA had a significant increase in $[\mathrm{HbO}]$ in several source-detector pairs in both lateral hemispheres, with very little change in the occipital region (Fig. 2a). OA had a greater change in [HbO] in most of the source-detector pairs bilaterally, and also had an increase in [HbO] in the occipital region (Fig. 2b). The region of interest (ROI) analysis confirmed that the changes from baseline to test were significant for all of the ROIs for both groups, except for the occipital and left frontal-lateral regions in the MA (Fig. 3A). Furthermore, the OA had significantly greater activation during the test compared with MA in the occipital $\left(t=7.4, p_{\mathrm{FDR}}<0.001\right)$, right temporal-parietal $\left(t=2.9, p_{\mathrm{FDR}}=0.014\right)$, and left frontal-lateral $\left(t=3.2, p_{\mathrm{FDR}}=0.006\right)$ ROIs.

During Trial 2, when the somatosensory information was degraded and visual input was not available, the OA and MA had similar patterns of increased $[\mathrm{HbO}]$ in most of the sourcedetector pairs in the anterior portion of the lateral hemispheres, but only the OA had increased [HbO] in the occipital region (Fig. 2c, d). The ROI analysis demonstrated significantly increased [HbO] for both groups in all regions, except the occipital region for MA (Fig. 3b). While the MA had activation that was slightly greater than OA in the lateral regions, it did not reach statistical significance. In contrast, OA had significantly greater activation compared with MA in the occipital ROI $\left(t=3.7, p_{\mathrm{FDR}}=0.001\right)$.

During Trial 3, when the somatosensory and vestibular inputs were accurate, and visual input was removed, the OA continued their pattern of a widespread increase in [HbO] in the lateral regions, while the MA had a relatively modest increase in just a few source-detector pairs of the lateral and occipital regions (Fig. 2e, f). Older adults produced significantly greater changes in activation compared with MA in the right frontal-lateral $\left(t=3.6, p_{\mathrm{FDR}}=\right.$ $0.002)$ and right temporal-parietal $\left(t=3.2, p_{\mathrm{FDR}}=0.006\right)$ ROIs (Fig. $\left.3 \mathrm{c}\right)$.

During Trial 4, when visual input was removed while somatosensory information was degraded throughout the trial, the MA had increased [HbO] in several channels of the left frontal-lateral region, and the right temporal-parietal region, and negligible increase in the occipital region (Fig. 2g). For the OA, the increase in [HbO] was consistent across all source-detector pairs in the lateral regions and there was a strong increase in the occipital region (Fig. 2h). The change in activation for both groups during the test condition was evident in all ROIs, except for the occipital ROI in MA (Fig. 3d). A significant difference was demonstrated between groups in the occipital $\left(t=5.0, p_{\mathrm{FDR}}<0.001\right)$ and right frontallateral $\left(t=4.9, p_{\mathrm{FDR}}<0.001\right)$ ROIs. 


\section{Postural sway}

The RMS center of pressure in the anteroposterior direction during the different trials is shown in Fig. 4. No between group effects were detected for any of the trials. A significant Block $\times$ Trial interaction was found $(p<0.001$ ), in which the test condition (Block B, swayreferenced platform) generated more COP than the baseline condition (Block $A_{1}$ and $A_{2}$, fixed platform), during both eyes open in the light (Trial 1) and eyes open in the dark (Trial 2) conditions. However, we did not observe significant changes in COP when participants transitioned to eyes open in the dark from eyes open in the light, on fixed (Trial 3) or swayreferenced (Trial 4) platform conditions.

\section{Discussion}

In this study, we used fNIRS to investigate cortical activity in middle-aged and older adults during multi-sensory integration postural control conditions. The primary findings were that the transition to more impoverished sensory input resulted in greater activity in almost every ROI of both groups, and that the older adults had greater activity in several regions of interest, but most consistently the occipital region. Overall, we propose that these changes in brain activation during actual balance tasks demonstrate a cortical contribution to the sensory re-weighting process. Furthermore, it is apparent that in older adults, sensory reweighting is more distributed and requires greater attention compared with middle-aged adults.

The trial-by-trial results have important implications with regard to understanding the cortical control of posture in older adults. During Trial 1, the reduced somatosensory input while retaining accurate visual and vestibular information resulted in greater activation in the occipital and right temporal-parietal ROIs in OA compared with MA, indicating that older adults maximized their visual and vestibular contributions to sensory integration, whereas the middle-aged adults did not appear to increase their visual contribution. In addition, the relative increase in the left frontal-lateral ROI in the OA compared with MA suggests that the increased effort devoted to the sensory integration may come at an increased attentional cost for OA.

In the test condition of Trial 2, accurate somatosensory input was degraded under the already existing condition of no visual input, meaning that only vestibular input could be used for control of standing. The OA had a significant increase in activation in the occipital ROI, whereas MA only had a small non-significant increase. These findings suggest that OA were attempting to use any available sensory information because of the impoverished sensory conditions, even information that was not available. On the other hand, there was no statistical difference in the amount of increase in activation in the other ROIs between the $\mathrm{OA}$ and MA, indicating that the sensory integration and attentional load were relatively equal between the groups. The findings for Trial 4, which also featured a transition to vestibular-only input, were similar for the occipital ROI. However, increased activation in the frontal-lateral ROI in Trial 4 for OA compared with MA may reflect an increased attentional load in the OA.

Exp Brain Res. Author manuscript; available in PMC 2017 July 03. 
During Trial 3, somatosensory and vestibular inputs were always available, and visual input was removed. Overall, this transition is easiest to make, as demonstrated by the postural sway measurements, which changed negligibly. The increase in brain activation was the least during this trial, although it was still significant in most ROIs. The OA had significantly greater activation in the right frontal-lateral and right temporal-parietal ROIs compared with the MA, suggesting somewhat larger sensory integration and attention loading for the older adults.

In other neuroimaging studies of posture and gait, older and younger adults have frequently demonstrated shared regions of activation as well as regions of different activation (Holtzer et al. 2014). For instance, an fMRI study of the motor imagery of walking shows consistent areas of activation in the supplementary motor area, caudate nucleus, visual cortex, and cerebellum among younger and older adults (Zwergal et al. 2012). The Zwergal study is noteworthy because it also showed that older adults had increased activity in the multisensory vestibular cortex (i.e. our temporal-parietal ROI) and motion-sensitive visual cortex (i.e. occipital ROI), compared with younger adults. Furthermore, increased task complexity produced greater activation in diffuse regions (Zwergal et al. 2012; Wai et al. 2012). Consequently, there appears to be a convergence of results favoring increased activation in multi-sensory areas during posture and gait tasks in older adults. The findings are also consistent with theories of cognitive aging that suggest that increased activation, especially bilaterally, is a result of compensation for declining cortical structures and function (Cabeza 2001; Papegaaij et al. 2014a), or a reduction in inhibitory function (Zwergal et al. 2012; Papegaaij et al. 2014b).

The brain activity during different sensory integration conditions has been studied in young adults ( $28 \pm 9$ years) by Karim et al. (2013a). The patterns of activation in the temporalparietal regions of the MA and OA in our study generally reflect the patterns of the younger adult cohort as observed in the previous study (Karim et al. 2013a). However, the activation of frontal-lateral regions varied among the younger adults, MA and OA. In the younger adults, the right frontal-lateral region was activated and the left frontal-lateral region showed a relative decrease in activation when both visual information and somatosensory information were degraded (Karim et al. 2013a). In our study, the MA and OA, both, demonstrated an increase in activity in the left frontal-lateral region when both visual information and somatosensory information were absent. The frontal-lateral region is related to the attentional cost during activities (Radel et al. 2016). The relative increase in activity in the left frontal-lateral region in the OA and MA compared with younger adults suggests an increased attentional cost for OA and MA compared with younger adults. Finally, the occipital cortical areas were not measured in the previous study, and thus we were not able to compare the brain activity with the younger adults (Karim et al. 2013a).

\section{Conclusion}

Older adults demonstrated increased activity compared with middle-aged adults during sensory integration conditions, with greater frequency in the occipital region, followed by the frontal-lateral (attention) and temporal-parietal (vestibular) regions. However, the increased brain activities did not lead to group differences in postural sway. These findings

Exp Brain Res. Author manuscript; available in PMC 2017 July 03. 
suggest that in older adults, the sensory re-weighting process requires more resources to compensate for the reduction in sensory input. Future research should focus on the sensory re-weighting process in different groups of subjects, such as people with peripheral neuropathy or people with vestibular disorders, to understand how the impaired sensory input for balance control affects the cortical sensory re-weighting process. Using fNIRS to measure brain activity may also help to understand the cortical contributions to dynamic balance and walking conditions.

\section{Acknowledgments}

The authors give special thanks to Anita Lieb for her help on this project. Grants: This work was supported by the Pennsylvania Lions Hearing Research Foundation (GA-3303), the University of Pittsburgh Older Americans Independence Center (NIH P30 AG024827), and the Eye and Ear Foundation.

\section{References}

Abdelnour AF, Huppert T. Real-time imaging of human brain function by near-infrared spectroscopy using an adaptive general linear model. Neuroimage. 2009; S1053-8119(09):00081-00087. doi: 10.1016/j.neuroimage.2009.01.033

Abdelnour F, Huppert T. A random-effects model for group-level analysis of diffuse optical brain imaging. Biomed Opt Express. 2010; 2:1-25. DOI: 10.1364/BOE.2.000001 [PubMed: 21326631]

Allum JH, Zamani F, Adkin AL, Ernst A. Differences between trunk sway characteristics on a foam support surface and on the Equitest ankle-sway-referenced support surface. Gait Posture. 2002; 16:264-270. [PubMed: 12443951]

Barker JW, Aarabi A, Huppert TJ. Autoregressive model based algorithm for correcting motion and serially correlated errors in fNIRS. Biomed Opt Express. 2013; 4:1366-1379. DOI: 10.1364/BOE. 4.001366189317 [PubMed: 24009999]

Boas DA, Dale AM, Franceschini MA. Diffuse optical imaging of brain activation: approaches to optimizing image sensitivity, resolution, and accuracy. Neuroimage. 2004; 23(Suppl 1):S275-S288. DOI: 10.1016/j.neuroimage.2004.07.011 [PubMed: 15501097]

Cabeza R. Cognitive neuroscience of aging: contributions of functional neuroimaging. Scand J Psychol. 2001; 42:277-286. [PubMed: 11501741]

Cham R, Perera S, Studenski SA, Bohnen NI. Striatal dopamine denervation and sensory integration for balance in middle-aged and older adults. Gait Posture. 2007; 26:516-525. DOI: 10.1016/ j.gaitpost.2006.11.204 [PubMed: 17196819]

Cope M, Delpy DT, Reynolds EO, Wray S, Wyatt J, van der Zee P. Methods of quantitating cerebral near infrared spectroscopy data. Adv Exp Med Biol. 1988; 222:183-189. [PubMed: 3129910]

Faraldo-Garcia A, Santos-Perez S, Crujeiras-Casais R, Labella-Caballero T, Soto-Varela A. Influence of age and gender in the sensory analysis of balance control. Eur Arch Otorhinolaryngol. 2012; 269:673-677. DOI: 10.1007/s00405-011-1707-7 [PubMed: 21789678]

Holmes CJ, et al. Enhancement of MR images using registration for signal averaging. J Comput Assist Tomogr. 1998; 22:324-333. [PubMed: 9530404]

Holtzer R, Epstein N, Mahoney JR, Izzetoglu M, Blumen HM. Neuroimaging of mobility in aging: a targeted review. J Gerontol A Biol Sci Med Sci. 2014; 69:1375-1388. DOI: 10.1093/gerona/ glu052 [PubMed: 24739495]

Huppert TJ, Hoge RD, Dale AM, Franceschini MA, Boas DA. Quantitative spatial comparison of diffuse optical imaging with blood oxygen level-dependent and arterial spin labeling-based functional magnetic resonance imaging. J Biomed Opt. 2006a; 11:064018.doi: 10.1117/1.2400910 [PubMed: 17212541]

Huppert TJ, Hoge RD, Diamond SG, Franceschini MA, Boas DA. A temporal comparison of BOLD, ASL, and NIRS hemodynamic responses to motor stimuli in adult humans. Neuroimage. 2006b; 29:368-382. DOI: 10.1016/j.neuroimage.2005.08.065 [PubMed: 16303317] 
Huppert TJ, Diamond SG, Franceschini MA, Boas DA. HomER: a review of time-series analysis methods for near-infrared spectroscopy of the brain. Appl Opt. 2009; 48:D280-D298. [PubMed: 19340120]

Huppert T, Schmidt B, Beluk N, Furman J, Sparto P. Measurement of brain activation during an upright stepping reaction task using functional near-infrared spectroscopy. Hum Brain Mapp. 2013; 34:2817-2828. DOI: 10.1002/hbm.22106 [PubMed: 23161494]

Jongkees LW. Value of the caloric test of the labyrinth. Arch Otolaryngol. 1948; 48:402-417. DOI: 10.1001/archotol.1948.00690040414003

Karim H, Schmidt B, Dart D, Beluk N, Huppert T. Functional near-infrared spectroscopy (fNIRS) of brain function during active balancing using a video game system. Gait Posture. 2012; 35:367372. DOI: 10.1016/j.gaitpost.2011.10.007S0966-6362(11)00411-5 [PubMed: 22078300]

Karim H, Fuhrman SI, Sparto P, Furman J, Huppert T. Functional brain imaging of multi-sensory vestibular processing during computerized dynamic posturography using near-infrared spectroscopy. Neuroimage. 2013a; 74:318-325. DOI: 10.1016/j.neuroimage. 2013.02.010S1053-8119(13)00137-7 [PubMed: 23419940]

Karim HT, Fuhrman SI, Furman JM, Huppert TJ. Neuroimaging to detect cortical projection of vestibular response to caloric stimulation in young and older adults using functional near-infrared spectroscopy (fNIRS). Neuroimage. 2013b; 76:1-10. DOI: 10.1016/j.neuroimage. 2013.02.061S1053-8119(13)00216-4 [PubMed: 23523804]

Laurienti PJ, Burdette JH, Maldjian JA, Wallace MT. Enhanced multisensory integration in older adults. Neurobiol Aging. 2006; 27:1155-1163. DOI: 10.1016/j.neurobiolaging.2005.05.024 [PubMed: 16039016]

Lee DN, Aronson E. Visual proprioceptie control of standing in human infants. Perception Psychophysics. 1974; 15:529-532.

Mahboobin A, Loughlin PJ, Redfern MS, Sparto PJ. Sensory re-weighting in human postural control during moving-scene perturbations. Exp Brain Res. 2005; 167:260-267. DOI: 10.1007/ s00221-005-0053-7 [PubMed: 16025292]

Mihara M, Miyai I, Hatakenaka M, Kubota K, Sakoda S. Role of the prefrontal cortex in human balance control. Neuroimage. 2008; 43:329-336. DOI: 10.1016/j.neuroimage. 2008.07.0291053-8119(08)00848-3 [PubMed: 18718542]

Nashner L, Berthoz A. Visual contribution to rapid motor responses during postural control. Brain Res. 1978; 150:403-407. [PubMed: 678978]

Nashner LM, Black FO, Wall C 3rd. Adaptation to altered support and visual conditions during stance: patients with vestibular deficits. J Neurosci. 1982; 2:536-544. [PubMed: 6978930]

O’Connor KW, Loughlin PJ, Redfern MS, Sparto PJ. Postural adaptations to repeated optic flow stimulation in older adults. Gait Posture. 2008; 28:385-391. DOI: 10.1016/j.gaitpost.2008.01.010 [PubMed: 18329878]

Papegaaij S, Taube W, Baudry S, Otten E, Hortobagyi T. Aging causes a reorganization of cortical and spinal control of posture. Front Aging Neurosci. 2014a; 6:28.doi: 10.3389/fnagi.2014.00028 [PubMed: 24624082]

Papegaaij S, Taube W, Hogenhout M, Baudry S, Hortobagyi T. Age-related decrease in motor cortical inhibition during standing under different sensory conditions. Front Aging Neurosci. 2014b; 6:126.doi: 10.3389/fnagi.2014.00126 [PubMed: 24971063]

Peterka RJ. Sensorimotor integration in human postural control. J Neurophysiol. 2002; 88:1097-1118. [PubMed: 12205132]

Peterka RJ, Loughlin PJ. Dynamic regulation of sensorimotor integration in human postural control. J Neurophysiol. 2004; 91:410-423. DOI: 10.1152/jn.00516.2003 [PubMed: 13679407]

Radel R, Brisswalter J, Perrey S. Saving mental effort to maintain physical effort: a shift of activity within the prefrontal cortex in anticipation of prolonged exercise. Cogn Affect Behav Neurosci. 2016; doi: 10.3758/s13415-016-0480-x

Taube W, Gruber M, Beck S, Faist M, Gollhofer A, Schubert M. Cortical and spinal adaptations induced by balance training: correlation between stance stability and corticospinal activation. Acta Physiol (Oxf). 2007; 189:347-358. DOI: 10.1111/j.1365-201X.2007.01665.x [PubMed: 17263693] 
Tsuzuki D, Dan I. Spatial registration for functional near-infrared spectroscopy: from channel position on the scalp to cortical location in individual and group analyses. Neuroimage. 2014; 85(Pt 1):92103. [PubMed: 23891905]

Wai YY, Wang JJ, Weng YH, Lin WY, Ma HK, Ng SH, Wan YL, Wang CH. Cortical involvement in a gait-related imagery task: comparison between Parkinson's disease and normal aging. Parkinsonism Relat Disord. 2012; 18:537-542. DOI: 10.1016/j.parkreldis.2012.02.004 [PubMed: 22436654]

Whitney SL, Marchetti GF, Schade A, Wrisley DM. The sensitivity and specificity of the Timed "Up \& Go" and the Dynamic Gait Index for self-reported falls in persons with vestibular disorders. J Vestib Res. 2004; 14:397-409. [PubMed: 15598995]

Woollacott MH, Shumway-Cook A. Changes in posture control across the life span-a systems approach. Phys Ther. 1990; 70:799-807. [PubMed: 2236223]

Woollacott M, Debu B, Mowatt M. Neuromuscular control of posture in the infant and child: is vision dominant? J Mot Behav. 1987; 19:167-186. [PubMed: 14988057]

Wrisley DM, Marchetti GF, Kuharsky DK, Whitney SL. Reliability, internal consistency, and validity of data obtained with the functional gait assessment. Phys Ther. 2004; 84:906-918. [PubMed: 15449976]

Ye JC, Tak S, Jang KE, Jung J, Jang J. NIRS-SPM: statistical parametric mapping for near-infrared spectroscopy. Neuroimage. 2009; 44:428-447. DOI: 10.1016/j.neuroimage. 2008.08.036S1053-8119(08)00969-5 [PubMed: 18848897]

Zwergal A, Linn J, Xiong G, Brandt T, Strupp M, Jahn K. Aging of human supraspinal locomotor and postural control in fMRI. Neurobiol Aging. 2012; 33:1073-1084. DOI: 10.1016/j.neurobiolaging. 2010.09.022 [PubMed: 21051105]

Exp Brain Res. Author manuscript; available in PMC 2017 July 03. 


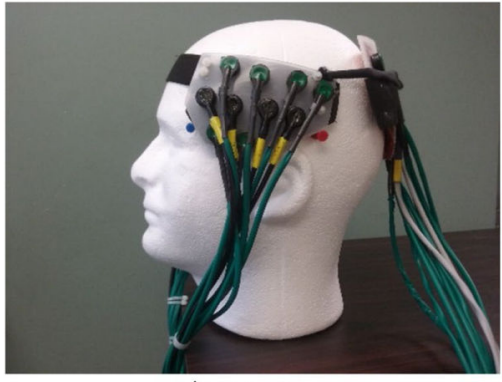

A Side view

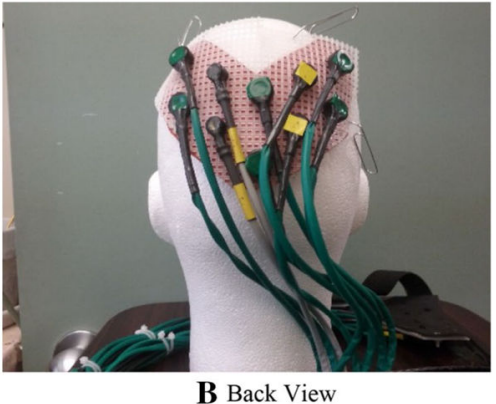

B Back View

Fig. 1.

Probe setting 


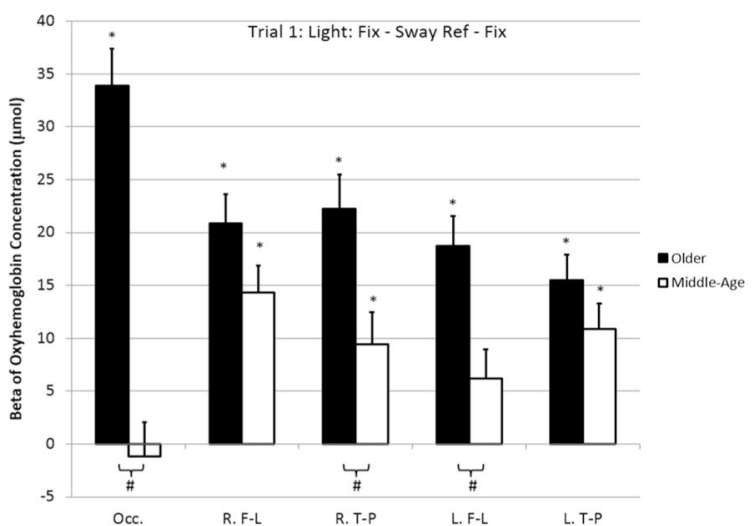

A

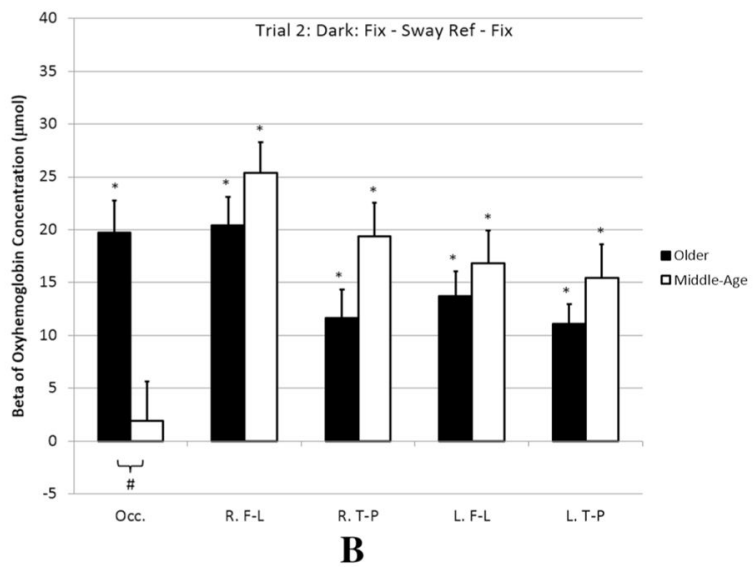

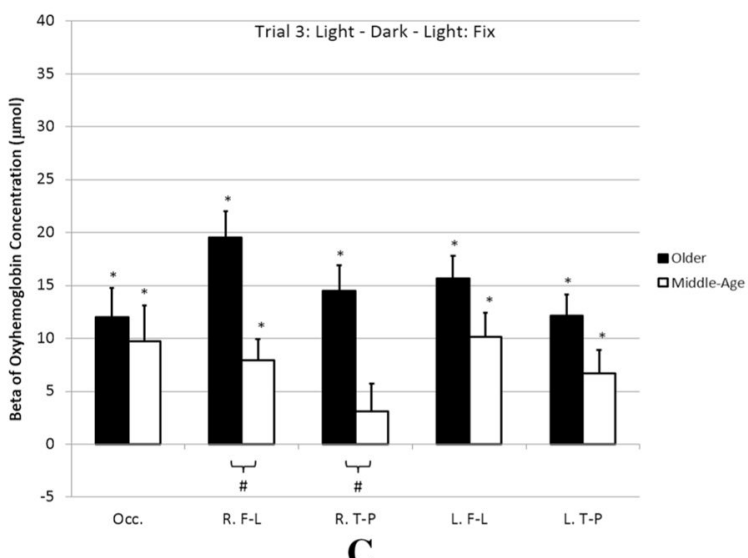

C

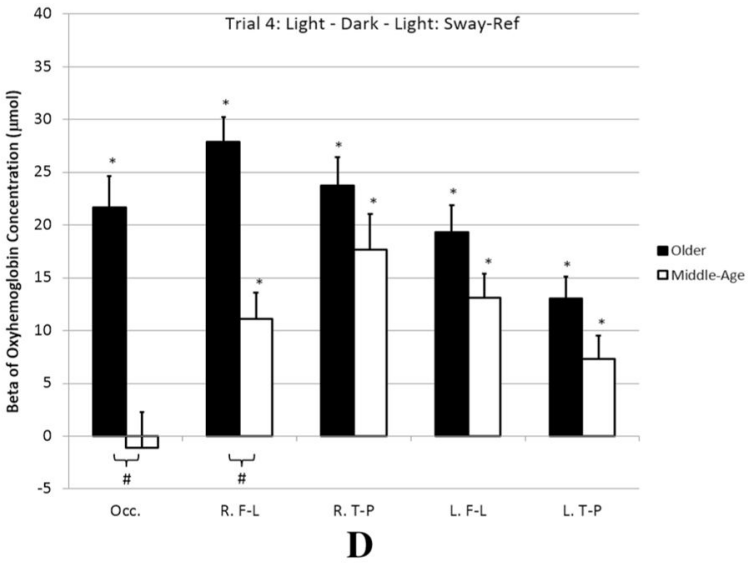

Fig. 2.

a, b Estimated regression coefficients, $\beta$, of changes in Oxy-Hemoglobin $\left(\mathrm{HbO}_{2}\right)$ from baseline conditions to test conditions in Older and Middle-age adults for Trial 1 (top) and Trial 2 (bottom). Regions of Interest Occipital (Occ), Right Fronto-lateral (R. F-L.), Right Temporo-Parietal (R. T-P), Left Fronto-lateral (L. F-L), and Left Temporo-Parietal (L. T-P). *Significant difference between task and baseline, $p_{\text {FDR }}<0.05$. ${ }^{*}$ Significant difference between Older and Middle-age adults, $p_{\mathrm{FDR}}<0.05$. c, $\mathbf{d}$ Estimated regression coefficients, $\beta$, of changes in Oxy-Hemoglobin $\left(\mathrm{HbO}_{2}\right)$ from baseline conditions to test conditions in Older and Middle-age adults for Trial 3 (top) and Trial 4 (bottom). Regions of Interest Occipital (Occ), Right Fronto-lateral (R. F-L.), Right Temporo-Parietal (R. T-P), Left Fronto-lateral (L. F-L), and Left Temporo-Parietal (L. T-P). *Significant difference between task and

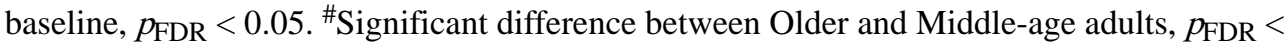
0.05 

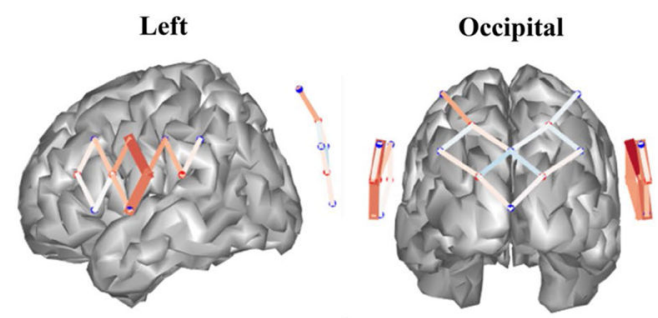

A Middle-Age Adults, Trial 1
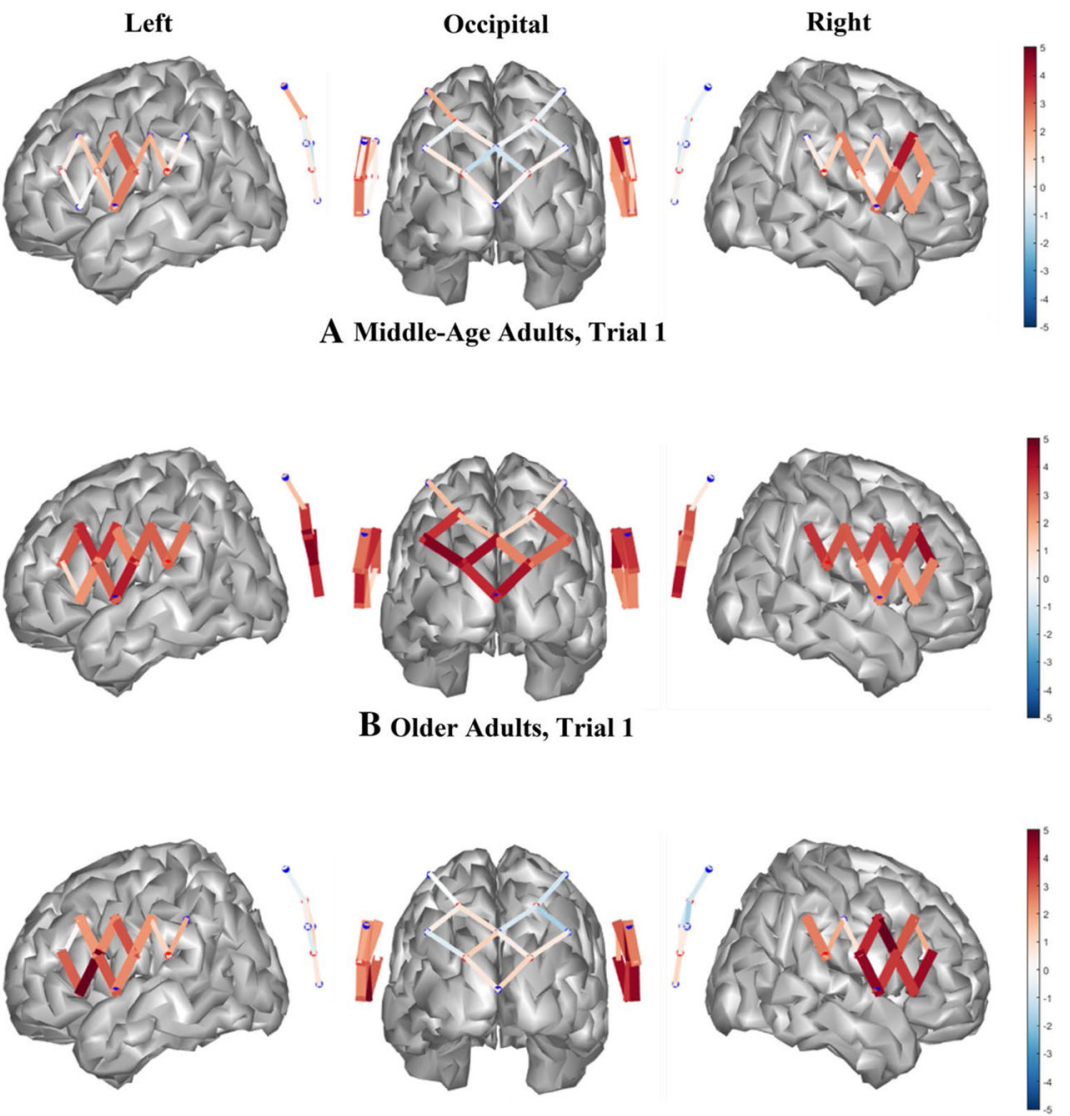

C Middle-Age Adults, Trial 2
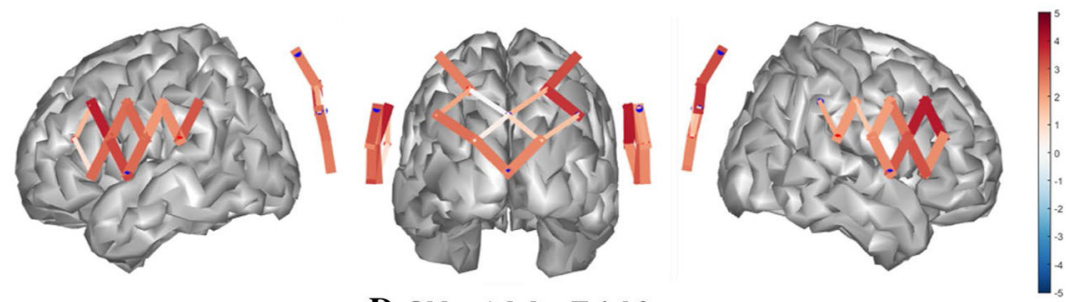

D Older Adults, Trial 2 

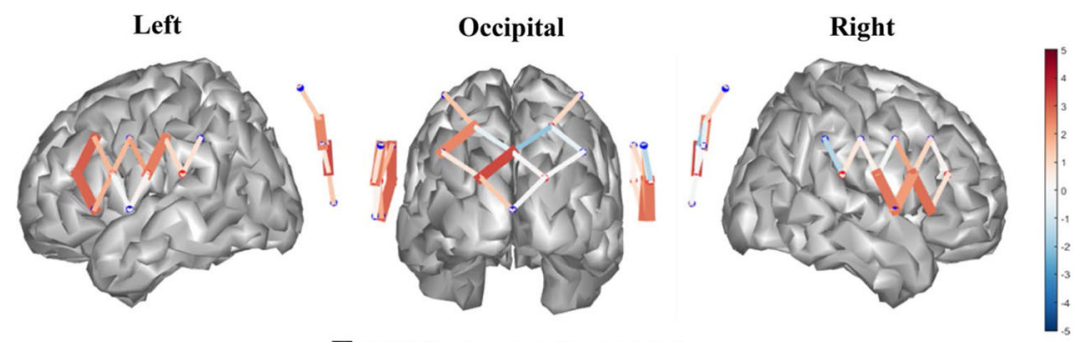

E Middle-Age Adults, Trial 3
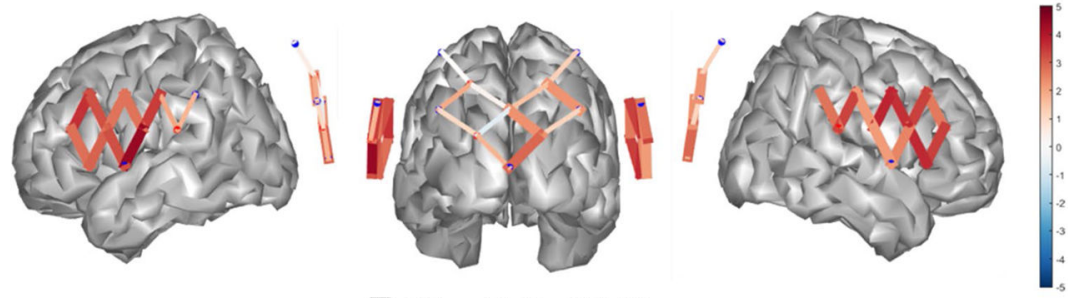

F Older Adults, Trial 3
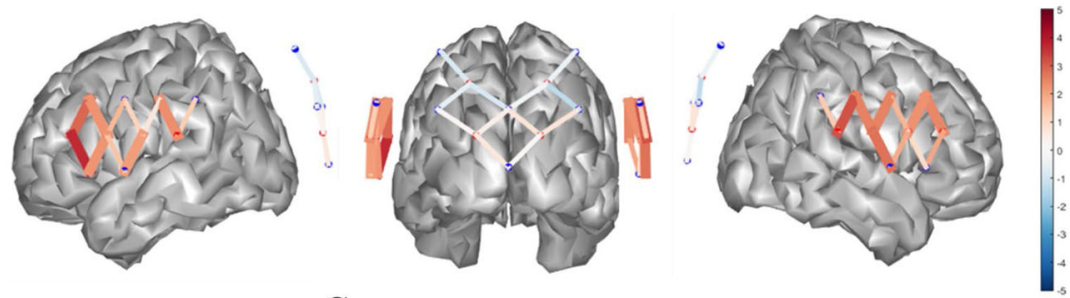

G Middle-Age Adults, Trial 4
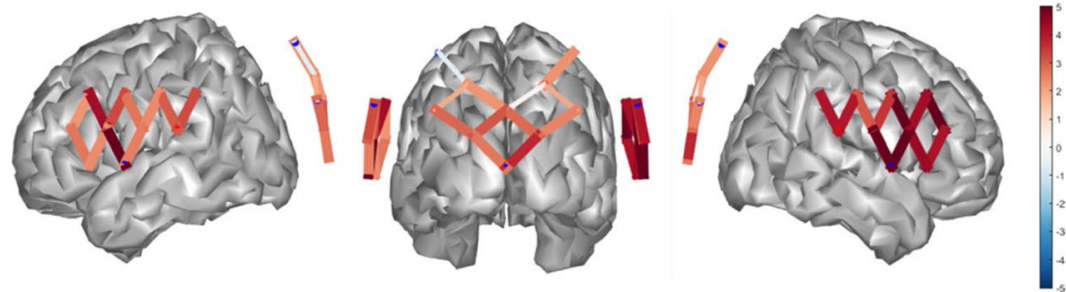

H Older Adults, Trial 4

Fig. 3.

a-d T-score maps of fNIRS oxyhemoglobin changes from baseline to test conditions for individual channels during the four different sensory integration trials in Middle-Aged (a, c, $\mathbf{e}, \mathbf{g})$ and Older $(\mathbf{b}, \mathbf{d}, \mathbf{f}, \mathbf{h})$ adults. Channels that were significantly activated $\left(p_{\mathrm{FDR}}<0.05\right)$ are shown as thick lines overlying brain. $\mathbf{e}, \mathbf{h}$ T-score maps of fNIRS oxyhemoglobin changes from baseline to test conditions for individual channels during the four different sensory integration trials in Middle-aged $(\mathbf{a}, \mathbf{c}, \mathbf{e}, \mathbf{g})$ and Older $(\mathbf{b}, \mathbf{d}, \mathbf{f}, \mathbf{h})$ adults. Channels that were significantly activated $\left(p_{\mathrm{FDR}}<0.05\right)$ are shown as thick lines overlying brain 
A Middle-Age Adults
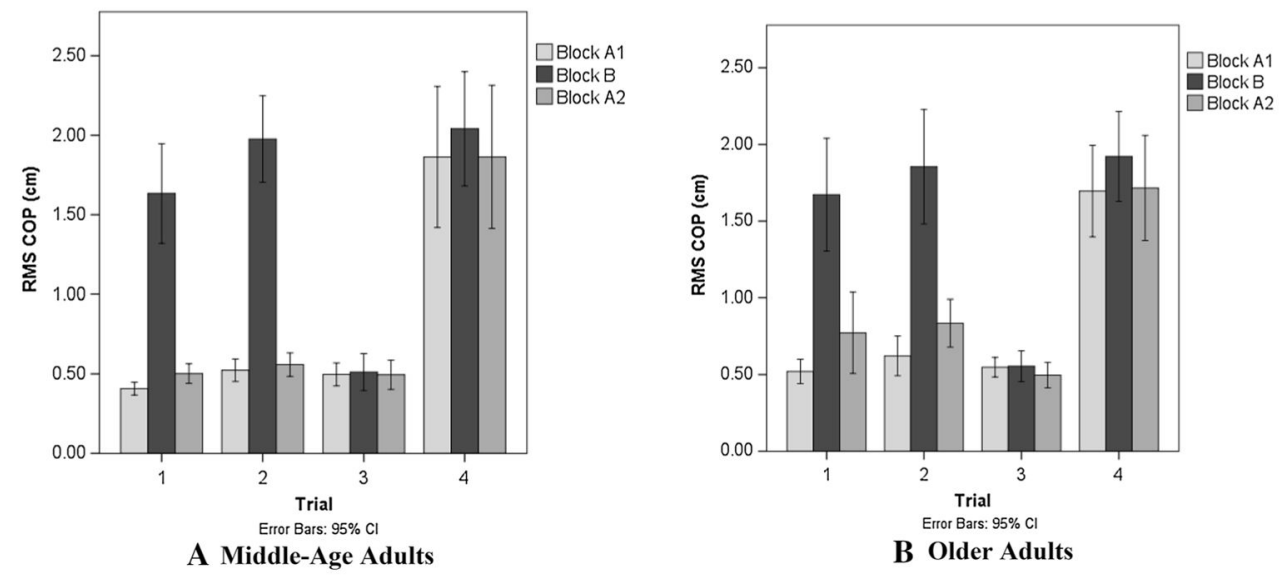

Fig. 4.

RMS COP in four different sensory integration trials in Middle-age (a) and Older (b) adults 


\section{Table 1}

Description of experimental trials. During each test block, the sensory input condition that changes is underlined

\begin{tabular}{lllll}
\hline Trial & Sensory feedback & Pre-baseline $\left(\mathbf{A}_{\mathbf{1}}\right)$ & Test $(\mathbf{B})$ & Post-baseline $\left(\mathbf{A}_{2}\right)$ \\
\hline 1 & Vision & Eyes open in light & Eyes open in light & Eyes open in light \\
& Somatosensation & Fixed platform & Sway-ref. platform & Fixed platform \\
& Avail. feedback & VIS, SS, VEST & VIS, VEST & VIS, SS, VEST \\
2 & Vision & Eyes open in dark & Eyes open in dark & Eyes open in dark \\
& Somatosensation & Fixed platform & Sway-ref. platform & Fixed platform \\
& Avail. feedback & SS, VEST & VEST & SS, VEST \\
3 & Vision & Eyes open in light & Eyes open in dark & Eyes open in light \\
& Somatosensation & Fixed platform & Fixed platform & Fixed platform \\
& Avail. feedback & VIS, SS, VEST & SS, VEST & VIS, SS, VEST \\
& Vision & Eyes open in light & Eyes open in dark & Eyes open in light \\
& Somatosensation & Sway-ref. platform & Sway-ref. platform & Sway-ref. platform \\
& Avail. feedback & VIS, VEST & VEST & VIS, VEST \\
\hline
\end{tabular}

$V I S$ accurate visual input, $S S$ accurate somatosensory input, VEST Accurate peripheral vestibular input 